\title{
Fatores associados a não adesão medicamentosa entre idosos de um ambulatório filantrópico do Espírito Santo
}

\author{
Pharmacological non-adherence therapy and associated factors among elderly from a philanthropic \\ outpatient unit of Espírito Santo state, Brazil
}

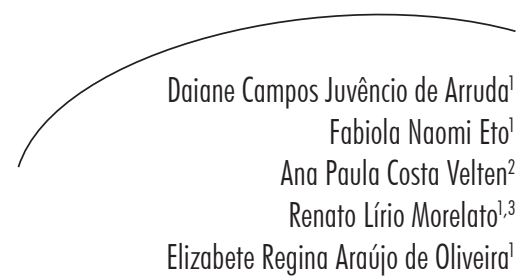

Resumo

Objetivos: Estimar a frequência da não adesão ao tratamento medicamentoso entre idosos acompanhados ambulatorialmente, bem como analisar seus fatores associados. Metodologia: Foi conduzido estudo transversal com 263 idosos atendidos no ambulatório de especialidades médicas de um hospital filantrópico, localizado no município de Vitória-ES. Foi realizada entrevista utilizando roteiro estruturado em três blocos que contemplavam questões sociodemográficas, condições de saúde e estilo de vida e medicamentos em uso. Para verificar a não adesão ao tratamento medicamentoso, foi aplicado o instrumento de Medida de Adesão Terapêutica (MAT). Os dados foram analisados por meio do teste Qui-quadrado para variáveis categóricas. Foram consideradas significativas as variáveis com valor de $p<0,05$ no modelo final de regressão múltipla de Poisson. Resultados: Os resultados mostraram uma frequência de não adesão ao tratamento medicamentoso de $26,7 \%$ da amostra. A não adesão ao tratamento medicamentoso nessa população se mostrou positivamente associada à ausência de vínculo empregatício anterior a aposentadoria $(\mathrm{RP}=1,12 ; p<0,010)$; presença de declínio cognitivo $(\mathrm{RP}=1,13 ; p<0,010)$ e hábitos alimentares inadequados $(\mathrm{RP}=1,12$; $p<0,005)$. Conclusão: Com o estudo, foi possível identificar os fatores associados à não adesão medicamentosa nos idosos investigados e assim contribuir para o conhecimento do perfil sociodemográfico, condições de saúde e estilo de vida e características relacionadas à utilização de medicamentos por parte dessa população.

\section{Abstract}

Objectives: To estimate the non-adherence frequency of drug treatment among elderly outpatients and to analyze associated factors. Methods: A cross-sectional study was conducted with 263 elderly patients from the medical specialties outpatient of a philanthropic hospital, located in the city of Vitoria, Espirito Santo state, Brazil.

Universidade Federal do Espírito Santo, Centro de Ciências da Saúde, Programa de Pós-graduação em Saúde Coletiva. Vitória, ES, Brasil.

2 Universidade Federal do Espírito Santo, Centro Universitário Norte do Espírito Santo, Departamento de Ciências da Saúde. São Mateus, ES, Brasil.

3 Escola Superior de Ciências da Santa Casa de Misericórdia de Vitória, Programa de Residência Médica de Geriatria. Vitória, ES, Brasil.

Palavras-chave: Adesão à Medicação. Idoso. Terapêutica. Assistência Ambulatorial. 
Interview was conducted using a structured script in three blocks that contemplated sociodemographic, health and lifestyle and medications used. To check the nonadherence to drug treatment, Measurement of Treatment Adherence (MTA) was applied. Data were analyzed using the chi-square test for categorical variables. The variables with $p<0.05$ in the final model of multiple Poisson regression were considered significant. Results: The results showed a frequency of non-adherence to drug treatment in $26.7 \%$ of the sample. Non-adherence to drug treatment in this population was positively associated with lack of employment prior to retirement ( $\mathrm{PR}=1.12 ; p<0.010)$; the presence of cognitive decline $(\mathrm{PR}=1.13 ; p<0.010)$ and poor eating habits $(\mathrm{PR}=1.12$; $p<0.005)$. Conclusion: With this study, it was possible to identify the factors associated with non-adherence to drug treatment in the investigated elderly and thus contribute to the knowledge of the socio-demographic profile, health and lifestyle and characteristics related to the use of drugs by this population.
Key words: Medication Adherence. Elderly. Therapeutics. Ambulatory Care.

\section{INTRODUÇÃo}

O tratamento medicamentoso é um dos fatores mais relevantes para a assistência à saúde em qualquer idade, especialmente em indivíduos idosos, que constituem cerca de 50\% dos usuários de fármacos. O elevado número de usuários da terceira idade ocorre em virtude da maior carga de doenças e incapacidades nessa faixa etária. ${ }^{1}$

Devido ao avanço da transição demográfica e epidemiológica no cenário global, evidenciase aumento da prevalência, bem como da manutenção das doenças crônico-degenerativas na população. Doenças crônicas acometem inúmeros idosos e requerem tratamentos prolongados, além do uso de diversos medicamentos (polifarmácia), o que pode gerar efeitos adversos, interações medicamentosas e erros de medicação, aumentando, dessa maneira, o risco de hospitalização. ${ }^{1}$ Dessa forma, tornase necessário o uso adequado dos fármacos prescritos, a fim de evitar os riscos advindos da não adesão medicamentosa, e garantir a efetividade do tratamento.

A adesão ao tratamento medicamentoso pode ser compreendida como a utilização, em pelo menos $80 \%$, do total dos medicamentos prescritos, observando fatores como horário, dose e duração do tratamento. O uso incorreto de medicamentos, a subutilização, o uso irracional ou não utilização total dos fármacos prescritos são formas de não adesão ao tratamento medicamentoso. ${ }^{2}$
Segundo a Organização Mundial da Saúde, ${ }^{3}$ a média de adesão ao tratamento para doenças crônicas em países desenvolvidos é de $50 \%$, sendo ainda menor em países em desenvolvimento. Com base em revisão sistemática ${ }^{4}$ realizada, foi verificado que a não adesão ao tratamento medicamentoso varia de 40 a 60\% na população geral. Esse índice é considerado elevado, visto que a não adesão à terapêutica acarreta complicações da doença, surgimento de novas patologias, hospitalizações e até mesmo a morte, promovendo elevados custos financeiros para o sistema de saúde. ${ }^{5}$

Como fatores de risco para não adesão aos fármacos nessa faixa etária, pode-se citar a complexidade dos esquemas terapêuticos, a falta de entendimento, o esquecimento devido ao comprometimento cognitivo, a diminuição da acuidade visual e da destreza manual nas atividades, a baixa escolaridade, o fato de residir sozinho, a polifarmácia e os efeitos adversos. ${ }^{6-9}$

Fatores relacionados ao estilo de vida, como hábitos alimentares, atividade física, tabagismo e alcoolismo interferem no estado de saúde física e mental do idoso e podem estar associados à adesão medicamentosa dessa população..$^{5-7}$ Tendo em vista os múltiplos fatores envolvidos, a não adesão aos fármacos prescritos não deve ser vista como um fenômeno isolado - antes, deve ser avaliada considerando o contexto das características individuais, sociodemográficas e estilo de vida; as condições de saúde e do tratamento; o processo de cuidado e a 
organização do sistema de saúde no qual o sujeito está inserido. 4,5

O conhecimento dos possíveis fatores associados à não adesão medicamentosa entre idosos contribuirá para a elaboração de estratégias em saúde voltadas para o aumento da adesão ao tratamento. Para tanto, este estudo objetivou estimar a frequência da não adesão ao tratamento medicamentoso entre idosos acompanhados ambulatorialmente e analisar seus fatores associados.

\section{METODOLOGIA}

Foi conduzido estudo transversal com idosos atendidos no ambulatório de especialidades médicas do Hospital Santa Casa de Misericórdia de Vitória (HSCMV), localizado no município de Vitória-ES.

Para participação dos idosos, foram adotados os seguintes critérios de inclusão: apresentar idade igual ou superior a 60 anos; residir no Estado do Espírito Santo; receber atendimento no ambulatório de endocrinologia ou cardiologia do HSCMV; estar em posse dos medicamentos, das embalagens ou da prescrição médica dos medicamentos atuais e possuir condições de responder às questões abordadas ou estar acompanhado de pessoas que pudessem responder ao questionário.

Para constituição da amostra, foi realizado o cálculo amostral com base no volume do fluxo de consultas médicas mensais do ambulatório de cardiologia e endocrinologia (por apresentarem fluxo elevado de atendimento de idosos) nos seis meses anteriores à data da coleta de dados (cerca de 600 pacientes por mês) e considerando a prevalência da adesão ao tratamento medicamentoso por idosos de 50\%. A margem de erro considerada para o cálculo foi de $5 \%$, num intervalo de confiança de 95\%. Dessa forma, a amostra calculada foi prevista para 235 idosos. A fim de suprir possíveis perdas ou recusas, foram acrescentados $10 \%$, resultando numa amostra de 258 sujeitos de pesquisa. Com base na listagem de consultas agendadas, os idosos foram contatados por telefone para levarem todos os medicamentos em uso no dia da consulta, tanto os fármacos prescritos (acompanhados da prescrição médica), como os não prescritos. Aos idosos não contatados anteriormente por telefone foram solicitados os medicamentos utilizados, as caixas e a prescrição médica dos mesmos no momento da entrevista.

A coleta de dados foi realizada por entrevistadores previamente treinados (em estudo piloto) e ocorreu em duas etapas, entre os meses de dezembro de 2011 a março de 2012.

Durante a primeira etapa da coleta de dados, foi realizada a avaliação cognitiva do idoso mediante aplicação do Miniexame do Estado Mental (MEEM), ${ }^{10,11}$ instrumento amplamente utilizado em pesquisas internacionais ${ }^{9,12} \mathrm{e}$ nacionais $^{13}$ para avaliação do estado cognitivo em pacientes geriátricos. A avaliação cognitiva dos idosos foi realizada como critério para que se pudesse prosseguir com a segunda parte da coleta de dados sem que houvesse comprometimento das informações a serem concedidas.

No presente estudo, foi considerado como ponto de corte o valor de 22 pontos obtidos no MEEM, ou seja, os idosos com menor avaliação cognitiva (até 21 pontos) foram considerados como apresentando comprometimento cognitivo. Para esses idosos, com avaliação inferior a 22 pontos, foi solicitada a colaboração do acompanhante para prosseguirem para a segunda etapa do estudo. Os idosos que atingiram no mínimo 22 pontos participaram da segunda etapa do estudo sem auxílio do acompanhante.

$\mathrm{Na}$ segunda etapa da coleta de dados, foi realizada entrevista por meio de roteiro estruturado em três blocos. No bloco 1, foram coletados dados de identificação; socioeconômicos e demográficos, que incluíram: gênero (feminino/ masculino); faixa etária (60-69 anos, 70-79 anos e 80 anos ou mais); raça (branca/não branca); escolaridade (não alfabetizado/alfabetizado); situação conjugal (com companheiro/sem companheiro); aposentadoria (sim/não); renda (até 1 salário mínimo, 2-3 salários mínimos e mais que 3 salários mínimos) e vínculo empregatício anterior (com vínculo/sem vínculo). 
No bloco 2, foram coletados dados relativos a condições de saúde e estilo de vida, incluindo as seguintes variáveis: declínio cognitivo (presente/ ausente); autoavaliação de saúde (boa/ruim); agravos pregressos (hipertensão arterial sistêmica [HAS], diabetes mellitus [DM], quedas); quantidade de agravos (1 a 2 doenças / 3 ou mais doenças); escala de independência em Atividade Básica de Vida Diária (ABVD), pelo índice de Katz ${ }^{14}$ (independência, dependência leve, dependência moderada); alcoolismo (presente [atual ou exalcoolista] / ausente [nunca]); tabagismo (presente [atual ou ex-tabagista] / ausente [nunca]); atividade física (ativo/sedentário) e hábitos alimentares (adequado/inadequado).

Por fim, no bloco 3 foram coletados dados referentes aos medicamentos utilizados no último mês e verificada a adesão ao tratamento por meio do Instrumento de Medida de Adesão ao Tratamento (MAT). ${ }^{15}$ As variáveis analisadas foram: polifarmácia, considerada como o consumo de cinco ou mais tipos de fármacos por dia (presente/ausente), e auxílio da administração de medicamentos (sem auxílio/com auxílio).

O MAT é composto por sete questões relacionadas com a adesão ao tratamento. Para cada pergunta, há seis opções de resposta (escala Likert) que variam entre "sempre" e "nunca". De acordo com o protocolo de pontuação do teste, é considerado aderente ao tratamento o indivíduo que obtém pontuação maior que 34 pontos na resposta às perguntas (sendo a numeração de cada resposta correspondente à pontuação da pergunta), e não aderente o que obtém até 34 pontos ao final das perguntas.

Para análise dos dados, foi considerada como variável dependente a não adesão ao tratamento medicamentoso. Os idosos foram agrupados conforme a seguinte classificação: indivíduos não aderentes ao tratamento medicamentoso e indivíduos aderentes ao tratamento medicamentoso. Para avaliação das diferenças entre os grupos de variáveis categóricas, foi realizado o teste qui-quadrado de Pearson.

As variáveis com nível de significância $\leq 0,20$ na análise bivariada foram incluídas no modelo de regressão múltipla de Poisson para análise das variáveis independentes associadas ao desfecho (RP bruta), ajustadas entre si e entre os possíveis fatores de confusão (RP ajustada) na análise múltipla.

Os dados coletados foram analisados utilizando o programa Statistical Package for the Social Sciences (SPSS) versão 22.0 (Série: 10101141221; Licença: fc48de7ce06356ade4c0).

O presente estudo seguiu as normas da Resolução no 196/96 do Conselho Nacional de Saúde; além disso, foi aprovado pelo Comitê de Ética em Pesquisa da Escola Superior de Ciências da Santa Casa de Misericórdia de Vitória (parecer $n^{\circ}$ 136/2011). Os idosos participaram do estudo voluntária e confidencialmente, após assinatura do Termo de Consentimento Livre e Esclarecido.

\section{RESULTADOS}

Foram selecionados e contatados 332 idosos, segundo os critérios de inclusão. Destes, foram excluídos 57 sujeitos por não portarem os medicamentos em uso ou a prescrição médica ou por apresentarem resultado MEEM menor que 22 pontos e não estarem acompanhados de pessoas que pudessem responder à entrevista. Assim, foram entrevistados 275 indivíduos, sendo 12 excluídos por apresentarem discordância nos dados coletados. A amostra final compreendeu 263 idosos, contemplando a amostra idealizada inicialmente. A frequência da não adesão medicamentosa nessa população foi de 26,7\% (tabela 1).

Quanto às variáveis sociodemográficas, observou-se que a maior frequência de não adesão ao tratamento medicamentoso foi entre idosos do gênero feminino $(77,6 \% ; p<0,42)$; na faixa etária de 60 a 69 anos $(54,3 \% ; p<0,40)$; não brancos $(67,1 \%$; $p<0,37)$; não alfabetizados $(34,3 \% ; p<0,19)$; que não possuíam companheiro $(57,2 \%$; $p<0,12)$; que eram aposentados $(52,9 \%$; $p<0,11)$; que apresentavam renda de até um salário mínimo $(70 \% ; p<0,99)$ e que não possuíam vínculo empregatício antes da aposentadoria $(62,9 \% ; p<0,01)$. 
Tabela 1. Frequência e associação bivariada da não adesão ao tratamento medicamentoso, segundo variáveis sociodemográficas, condições de saúde e estilo de vida e medicamentos utilizados, em idosos em acompanhamento ambulatorial. Vitória-ES, 2012.

\begin{tabular}{|c|c|c|c|c|c|c|}
\hline \multicolumn{2}{|c|}{ Variáveis sociodemográficas } & \multicolumn{2}{|c|}{$\begin{array}{c}\text { Não Aderente } \\
\qquad \begin{array}{c}n=70 \\
(26,7 \%)\end{array}\end{array}$} & \multicolumn{2}{|c|}{$\begin{array}{c}\text { Aderente } \\
n=193 \\
(73,3 \%)\end{array}$} & \multirow[t]{2}{*}{$p$-valor } \\
\hline & & $\mathrm{n}$ & $\%$ & $\mathrm{n}$ & $\%$ & \\
\hline \multirow{2}{*}{ Gênero } & Feminino & 55 & 28,0 & 141 & 72,0 & \multirow{2}{*}{0,42} \\
\hline & Masculino & 15 & 22,4 & 52 & 77,6 & \\
\hline \multirow{3}{*}{ Faixa etária } & 60-69 anos & 38 & 54,3 & 122 & 63,2 & \multirow{3}{*}{0,40} \\
\hline & 70-79 anos & 25 & 35,7 & 54 & 28,0 & \\
\hline & 80 anos ou mais & 7 & 10,0 & 17 & 8,8 & \\
\hline \multirow{2}{*}{ Raça } & Branca & 23 & 32,9 & 75 & 38,9 & \multirow{2}{*}{0,37} \\
\hline & Não branca & 47 & 67,1 & 118 & 61,1 & \\
\hline \multirow{2}{*}{ Escolaridade } & Não alfabetizado & 24 & 34,3 & 50 & 25,9 & \multirow{2}{*}{$0,19 *$} \\
\hline & Alfabetizado & 46 & 65,7 & 141 & 73,1 & \\
\hline \multirow{2}{*}{ Situação conjugal } & Com companheiro & 30 & 42,8 & 105 & 54,4 & \multirow{2}{*}{$0,12^{*}$} \\
\hline & Sem companheiro & 40 & 57,2 & 88 & 45,6 & \\
\hline \multirow{2}{*}{ Aposentadoria } & Sim & 37 & 52,9 & 123 & 63,7 & \multirow{2}{*}{$0,11^{*}$} \\
\hline & Não & 33 & 47,1 & 69 & 35,8 & \\
\hline \multirow{3}{*}{ Renda } & Até 1 salário mínimo & 49 & 70,0 & 134 & 69,4 & \multirow{3}{*}{0,99} \\
\hline & 2-3 salários mínimos & 17 & 24,3 & 49 & 25,4 & \\
\hline & Mais de 3 salários mínimos & 3 & 4,3 & 10 & 5,2 & \\
\hline \multirow{2}{*}{$\begin{array}{l}\text { Vínculo } \\
\text { empregatício } \\
\text { anterior }\end{array}$} & Com vínculo & 26 & 37,1 & 108 & 56,0 & \multirow[b]{2}{*}{$0,01 *$} \\
\hline & Sem vínculo & 44 & 62,9 & 84 & 43,5 & \\
\hline \multicolumn{6}{|c|}{$\begin{array}{l}\text { Variáveis relacionadas a condições } \\
\text { de saúde e estilo de vida }\end{array}$} & \\
\hline \multirow{2}{*}{ Declínio cognitivo } & Presente & 13 & 18,6 & 55 & 28,5 & \multirow{2}{*}{$0,16^{*}$} \\
\hline & Ausente & 57 & 81,4 & 138 & 71,5 & \\
\hline \multirow[b]{2}{*}{$\begin{array}{l}\text { Autoavaliação de } \\
\text { saúde }\end{array}$} & Boa & 26 & 37,1 & 99 & 51,3 & \multirow[b]{2}{*}{$0,05^{*}$} \\
\hline & Ruim & 44 & 62,9 & 94 & 48,7 & \\
\hline \multirow{3}{*}{$\begin{array}{l}\text { Agravos } \\
\text { pregressos** }\end{array}$} & HAS & 61 & 87,1 & 167 & 86,5 & 1,00 \\
\hline & $\mathrm{DM}$ & 28 & 40,0 & 90 & 46,6 & 0,40 \\
\hline & Quedas & 25 & 35,7 & 53 & 27,6 & 0,22 \\
\hline \multirow{2}{*}{$\begin{array}{l}\text { Quantidade de } \\
\text { agravos }\end{array}$} & 1 a 2 doenças & 13 & 18,6 & 44 & 22,8 & \multirow{2}{*}{0,50} \\
\hline & 3 ou mais doenças & 57 & 81,4 & 149 & 77,2 & \\
\hline
\end{tabular}




\begin{tabular}{|c|c|c|c|c|c|c|}
\hline \multirow{3}{*}{$\begin{array}{l}\text { Atividades básicas } \\
\text { da vida diária }\end{array}$} & Independência & 40 & 57,2 & 118 & 61,1 & \multirow{3}{*}{0,57} \\
\hline & Dependência leve & 29 & 41,4 & 73 & 37,8 & \\
\hline & Dependência moderada & 1 & 1,4 & 2 & 1,1 & \\
\hline \multirow[t]{2}{*}{ Tabagismo } & Presente (atual ou ex- fumante) & 24 & 34,3 & 67 & 34,7 & \multirow{2}{*}{0,999} \\
\hline & Ausente (nunca) & 46 & 65,7 & 123 & 63,7 & \\
\hline \multirow[t]{2}{*}{ Alcoolismo } & Presente (atual ou ex- alcoolista) & 24 & 34,3 & 67 & 34,7 & \multirow{2}{*}{0,26} \\
\hline & Ausente (nunca) & 46 & 65,7 & 126 & 65,3 & \\
\hline \multirow[t]{2}{*}{ Atividade física } & Ativo & 18 & 25,7 & 79 & 40,9 & \multirow{2}{*}{$0,03 *$} \\
\hline & Sedentário & 52 & 74,3 & 113 & 58,5 & \\
\hline \multirow{2}{*}{ Hábitos alimentares } & Adequado & 26 & 37,1 & 115 & 59,6 & \multirow{2}{*}{$0,001 *$} \\
\hline & Inadequado & 44 & 62,9 & 78 & 40,4 & \\
\hline \multicolumn{7}{|c|}{$\begin{array}{l}\text { Variáveis relacionadas aos } \\
\text { medicamentos utilizados }\end{array}$} \\
\hline \multirow[t]{2}{*}{ Polifarmácia $(\geq 5)$} & Presente & 43 & 61,4 & 111 & 57,5 & \multirow{2}{*}{0,57} \\
\hline & Ausente & 27 & 38,6 & 82 & 42,5 & \\
\hline \multirow{2}{*}{$\begin{array}{l}\text { Auxílio na } \\
\text { administração de } \\
\text { medicamentos }\end{array}$} & Sem auxílio & 54 & 77,1 & 164 & 85,0 & \\
\hline & Com auxílio & 16 & 22,9 & 29 & 15,0 & $0,14^{*}$ \\
\hline
\end{tabular}

*Variáveis cujo $p$-valor foi considerado significativo $(p<0,20)$ na análise bivariada e entraram no modelo de regressão múltipla; **idosos que podem ter sofrido mais de um agravo; HAS= hipertensão arterial sistêmica; $\mathrm{DM}=$ diabetes mellitus.

Em relação às variáveis relacionadas às condições de saúde e estilo de vida, predominaram como não aderentes ao tratamento medicamentoso os idosos com ausência de declínio cognitivo $(81,4 \% ; p<0,16)$; que autoavaliaram a saúde como "ruim" (62,9\%; $p<0,05)$ e que apresentavam independência nas atividades básicas da vida diária $(57,2 \% ; p<0,57)$

Quanto aos agravos e doenças pregressas autorreferidas, $87,1 \%$ dos participantes não aderentes apresentavam hipertensão arterial sistêmica $(p<1,00) ; 40 \%$, diabetes mellitus $(p<0,40)$ e cerca de $36 \%$ sofreram pelo menos um episódio de queda no último ano $(p<0,22)$.

Destaca-se a não adesão medicamentosa entre idosos que não fumavam ou nunca fumaram $(65,7 \% ; p<0,99)$ e que não ingeriam ou nunca ingeriram bebida alcoólica $(65,7 \% ; p<0,26)$. A maioria dos não aderentes era sedentária $(74,3 \%$; $p<0,03)$ e apresentava hábitos alimentares inadequados $(62,9 \% ; p<0,001)$.
Quanto ao uso dos medicamentos, a maioria dos idosos $(61,4 \% ; p<0,57)$ que não aderiam ao tratamento medicamentoso apresentaram polifarmácia e não recebiam auxílio na administração de medicamentos $(77,1 \%$; $p<0,14)$.

Foram incluídas na análise de regressão múltipla hierarquizada, bruta e ajustada todas as variáveis que apresentaram valor de $p<0,20$ na análise bivariada (tabela 2). Permaneceram associadas à não adesão medicamentosa na população estudada as variáveis que apresentaram valor de $p<0,05$. Dessa forma, foi verificado que os idosos sem vínculo empregatício apresentaram associação positiva $(\mathrm{RP}=1,12 ; \quad \mathrm{IC} 95 \%=1,02-1,23 ; \quad p<0,010)$ para a ocorrência de não adesão ao tratamento medicamentoso em relação aos que possuíam algum tipo de vínculo empregatício. Os idosos com declínio cognitivo apresentaram associação positiva $(\mathrm{RP}=1,13$; IC95\% $\%=1,03-1,21 ; p<0,010)$ para não adesão ao tratamento medicamentoso, 
quando comparados com aqueles que não apresentavam declínio cognitivo. Além disso, aqueles com hábitos alimentares considerados insatisfatórios também apresentaram associação positiva ( $\mathrm{RP}=1,12 ; \mathrm{IC} 95 \%=1,03-1,21 ; p<0,005)$ para não adesão ao tratamento medicamentoso, quando comparados àqueles com hábitos alimentares satisfatórios.

Tabela 2. Análise do modelo de regressão múltipla de Poisson bruta e ajustada para não adesão ao tratamento medicamentoso em idosos em acompanhamento ambulatorial. Vitória-ES, 2012.

\begin{tabular}{|c|c|c|c|c|c|}
\hline Variáveis & $\%$ & $\begin{array}{l}\mathrm{RP}(\mathrm{IC} 95 \%) \\
\text { bruta }\end{array}$ & $p^{*}$ & $\begin{array}{l}\text { RP (IC95\%) } \\
\text { ajustada }\end{array}$ & $p$ \\
\hline \multicolumn{6}{|l|}{ Escolaridade } \\
\hline Alfabetizado & 28,4 & 1 & & 1 & \\
\hline Não alfabetizado & 71,6 & $0,94(0,85-1,03)$ & 0,20 & $1,03(0,93-1,13)$ & 0,55 \\
\hline \multicolumn{6}{|l|}{ Situação conjugal } \\
\hline Com companheiro & 51,3 & 1 & & 1 & \\
\hline Sem companheiro & 48,7 & $0,93(0,85-1,01)$ & 0,09 & $0,92(0,85-1,00)$ & 0,052 \\
\hline \multicolumn{6}{|l|}{ Aposentadoria } \\
\hline $\operatorname{Sim}$ & 61,1 & 1 & & 1 & \\
\hline Não & 38,9 & $0,93(0,85-1,01)$ & 0,10 & $1,00(0,92-1,10)$ & 0,84 \\
\hline \multicolumn{6}{|l|}{$\begin{array}{l}\text { Vínculo empregatício } \\
\text { anterior }\end{array}$} \\
\hline Com vínculo & 51,1 & 1 & & 1 & \\
\hline Sem vínculo & 48,9 & $1,12(1,03-1,22)$ & 0,005 & $1,12(1,02-1,23)$ & $0,010^{*}$ \\
\hline \multicolumn{6}{|l|}{ Declínio cognitivo } \\
\hline Ausente & 74,1 & 1 & & 1 & \\
\hline Presente & 25,9 & $1,08(0,98-1,19)$ & 0,08 & $1,13(1,03-1,21)$ & $0,010^{*}$ \\
\hline \multicolumn{6}{|l|}{ Autoavaliação de saúde } \\
\hline Boa & 52,7 & 1 & & 1 & \\
\hline Ruim & 47,3 & $1,09(1,00-1,18)$ & 0,043 & $1,07(0,99-1,16)$ & 0,07 \\
\hline \multicolumn{6}{|l|}{ Atividade física } \\
\hline Ativo & 37,0 & 1 & & 1 & \\
\hline Sedentário & 63,0 & $1,10(1,01-1,20)$ & 0,01 & $1,07(0,98-1,17)$ & 0,086 \\
\hline \multicolumn{6}{|l|}{ Hábitos alimentares } \\
\hline Adequado & 53,6 & 1 & & 1 & \\
\hline Inadequado & 46,4 & $1,14(1,05-1,24)$ & 0,001 & $1,12(1,03-1,21)$ & $0,005^{*}$ \\
\hline \multicolumn{6}{|l|}{$\begin{array}{l}\text { Auxílio na administração } \\
\text { de medicamentos }\end{array}$} \\
\hline Sem auxílio & 17,1 & 1 & & 1 & \\
\hline Com auxílio & 82,9 & $1,08(0,97-1,21)$ & 0,15 & $1,01(0,98-1,24)$ & 0,087 \\
\hline
\end{tabular}

*Variáveis significativas $(p<0,05)$ na razão de prevalência $(\mathrm{RP})$ ajustada. 


\section{DISCUSSÃO}

O perfil sociodemográfico dos participantes deste estudo mostrou o predomínio do gênero feminino, na faixa etária de 60 a 69 anos, semelhante aos estudos de Block et al. ${ }^{16}$ e de Bastos-Barbosa et al., ${ }^{17}$ que também avaliaram a adesão ao tratamento medicamentoso em usuários de serviços públicos de saúde.

No presente estudo, a utilização de medicamentos foi mais frequente entre mulheres; apesar disso, elas foram menos aderentes, conforme apontam outras investigações epidemiológicas. ${ }^{9,18,19}$ Segundo informações da Pesquisa Nacional de Domicílios, ${ }^{20}$ as mulheres procuram mais por consultas médicas $(83,4 \%)$ em relação aos homens $(71,2 \%)$. Esse fato pode ser explicado pela maior prevalência e incidência de doenças crônicas no sexo feminino e devido ao comportamento de busca por cuidados de saúde ser mais frequente entre mulheres. ${ }^{20}$ Neste estudo, não houve associação estatística entre gênero e idade com a não adesão medicamentosa.

A média de consumo por dia de medicamentos foi de 5,4 fármacos por idoso, uma condição de polifarmácia em 58,6\% da amostra - média maior que a encontrada por Galato et al., ${ }^{21}$ de 3,5 medicamentos; Flores \& Mengue, ${ }^{18}$ de três a cinco; e por Loyola Filho et al., ${ }^{19}$ de 2,2 fármacos por idoso.

A frequência de não adesão ao tratamento medicamentoso na população estudada foi de $26,7 \%$, e os fatores significativamente associados a essa condição foram a ausência de vínculo empregatício, o declínio cognitivo e hábitos alimentares inadequados. Tal frequência é maior do que a encontrada por Faria ${ }^{22}(17,6 \%)$ e menor do que a encontrada por Rocha et al..$^{23}(63 \%)$.

Henriques, ${ }^{24}$ em revisão sistemática sobre a adesão à terapêutica em idosos, evidenciou que a proporção de adesão foi de $50 \%$. Carvalho et al., ${ }^{25}$ por meio da aplicação de dois instrumentos diferentes, encontraram adesão à farmacoterapia de $22,5 \%$ e $30,7 \%$, em hipertensos e diabéticos, respectivamente.
As diferenças entre as frequências encontradas podem ser atribuídas ao delineamento dos estudos e às diferentes metodologias adotadas de aferição da não adesão. Apesar da elevada frequência de adesão ao tratamento medicamentoso encontrada na população investigada ( $73,3 \%)$, esse número ainda se encontra abaixo do recomendado pela Organização Mundial da Saúde (80\%). ${ }^{3}$

A não adesão medicamentosa é mais frequente no nível da atenção primária do que entre pacientes de serviços especializados. ${ }^{23,26} \mathrm{Na}$ atenção básica primária, os idosos com maior risco, ou seja, aqueles que não aderem ao tratamento e que podem apresentar intercorrências em relação ao estado de saúde, são identificados e encaminhados ao tratamento ambulatorial especializado para melhor acompanhamento desse paciente.

A população do presente estudo é acompanhada pelos profissionais de saúde dos ambulatórios de especialidades endocrinológicas e cardiológicas do HSCMV. O atendimento a esses pacientes perpassa uma equipe de saúde multidisciplinar, sendo realizadas as consultas médica e de enfermagem, e o acompanhamento do assistente social, quando necessário. São disponibilizados os medicamentos quando o paciente não consegue adquirir na rede pública ou por recursos próprios, fator que, aparentemente, ajudaria na redução da frequência de não adesão ao tratamento. Vale ressaltar que, por se tratar de um hospital-escola, ele se constitui como referência nas consultas médicas e orientações adequadas ao paciente quanto ao uso dos medicamentos, que são realizadas pelos alunos sob supervisão do professor.

No estudo, a ausência de vínculo empregatício anterior à aposentadoria se mostrou como fator de risco (12\% maior) para não adesão ao tratamento medicamentoso por parte dos idosos. Não há evidências na literatura que mostrem associação entre a ausência de vínculo empregatício e não adesão ao tratamento medicamentoso em idosos. Entretanto, observou-se que idosos que não apresentavam nenhum tipo de vínculo empregatício anterior à aposentadoria foram considerados inativos profissionalmente, em 
razão de alguma limitação física, psíquica ou educacional, além da influência de demais fatores sociodemográficos.

Sabe-se que, em adultos, as desigualdades em saúde exercem importante influência sobre sua inserção no mercado de trabalho. Em relação à população idosa, provavelmente essa influência é mais acentuada, já que o envelhecimento é caracterizado pelo aumento da prevalência de doenças crônicas e maior utilização dos serviços de saúde. ${ }^{27}$

Estudo $^{27}$ que utilizou dados da Pesquisa Nacional por Amostra de Domicílios mostrou que, entre os idosos que trabalhavam, havia maior proporção de indivíduos que não relataram dificuldade para realização das atividades indicadoras de autonomia e mobilidade física, fator que provavelmente favorece a permanência dos mesmos no mercado de trabalho.

No presente estudo, cerca de $60 \%$ dos idosos utilizavam mais de cinco medicamentos - média de 5,4 fármacos por idoso. Em virtude da elevada incidência de doenças crônicas em idosos, o uso de múltiplos medicamentos é uma prática frequente que, além de ser complexa, exige maior atenção, memorização e organização para que haja adesão satisfatória ao tratamento medicamentoso. ${ }^{15,18}$

A presença de declínio cognitivo foi verificada, neste estudo, em cerca de 19\% dos idosos que não aderiram ao tratamento medicamentoso, sendo que aqueles com declínio cognitivo apresentaram risco 13\% maior de não aderir ao tratamento. Com o processo natural de envelhecimento, o declínio cognitivo se destaca como fator limitador para administração e adesão medicamentosa, uma vez que a adesão adequada ao tratamento das doenças incidentes nessa faixa etária pode ser desfavorecida em razão do déficit cognitivo e baixo nível de escolaridade, indicador sociodemográfico também observado na população do estudo.

Em razão das limitações funcionais que modificam os aspectos físicos e cognitivos do idoso, a prática de atividades de autocuidado relacionadas à saúde torna-se deficiente. Entre as práticas relacionadas ao autocuidado, está a adoção de hábitos saudáveis de estilo de vida - por exemplo, hábitos alimentares adequados e prática de atividades físicas -, promovendo melhores condições de saúde. A própria adesão ao tratamento é um dos componentes de autocuidado relacionado à saúde.

Na população investigada, 63\% dos idosos que não aderiam ao tratamento medicamentoso apresentaram hábitos alimentares inadequados. Foi encontrada associação de risco $(12 \%$ mais elevada) entre alimentação inadequada e a não adesão ao tratamento medicamentoso por parte desses idosos.

Estudo $^{28}$ realizado com idosos usuários de uma unidade básica de saúde, em João Pessoa$\mathrm{PB}$, mostrou que alguns idosos, mesmo tendo recebido orientações sobre a importância da alimentação saudável para redução da pressão arterial, não aderiam a hábitos alimentares satisfatórios. Muitos dos idosos que aderiam ao tratamento medicamentoso relataram não aderir a nenhum outro tipo de cuidado com a saúde, mesmo sabendo da importância de outras medidas. Entre as dificuldades relatadas pelos idosos não aderentes ao tratamento, estava seguir a dieta e mudar seu estilo de vida e, principalmente, a falta de instrução quanto ao tratamento medicamentoso e não medicamentoso.

Girotto et al. ${ }^{29}$ realizaram estudo com hipertensos cadastrados em uma unidade de saúde da família de Londrina-PR. Cerca de 70\% dos usuários referiram mudanças nos hábitos alimentares, sendo que se mostraram mais aderentes ao tratamento farmacológico aqueles que relataram mudanças na alimentação para controle da pressão arterial (63\%).

Em relação às limitações do presente estudo, destaca-se a utilização de informações autorreferidas, que podem ser influenciadas por viés de memória e levar à superestimação de alguns dados - por exemplo, a frequência de adesão ao tratamento. Entre outras limitações, 
cita-se a seleção da amostra por conveniência, fator que limita a extrapolação dos dados encontrados para outras populações e contextos.

\section{CONCLUSÃO}

Com os resultados obtidos, foi possível estimar a frequência da não adesão ao tratamento medicamentoso entre os idosos acompanhados ambulatorialmente. Além disso, a identificação e análise dos fatores associados à não adesão medicamentosa nos idosos investigados contribuíram para o conhecimento do perfil sociodemográfico, condições de saúde, estilo de vida e características relacionadas à utilização de medicamentos por parte dessa população. Com a elucidação desses fatores, torna-se possível a elaboração de estratégias direcionadas a populações com características

\section{REFERÊNCIAS}

1. Organização Pan-Americana da Saúde. Envelhecimento ativo: uma política de saúde. Brasília: OPAS; 2005 [acesso em 5 fev 2014]. Disponível em: http://bvsms.saude.gov.br/bvs/publicacoes/ envelhecimento_ativo.pdf.

2. Leite $\mathrm{SN}$, Vasconcellos MPC. Adesão à terapêutica medicamentosa: elementos para a discussão de conceitos e pressupostos adotados na literatura. Ciênc Saúde Coletiva 2003;8(3):775-82.

3. World Health Organization. Adherence to long term-therapies: evidence for action. Geneva: WHO; 2003 [acesso em 5 fev 2014]. Chapter 5, Towards the solution; 27-38. Disponível em: http://whqlibdoc. who.int/publications/2003/9241545992.pdf.

4. Higgins N, Regan C. A systematic review of the effectiveness of interventions to help older people adhere to medication regimes. Age Ageing 2004;33(3):224-9.

5. Gellad WF, Grenard JL, Marcum ZA. A systematic review of barriers to medication adherence in the elderly: looking beyond cost and regimen complexity. Am J Geriatr Pharmacother 2011;9(1):11-23.

6. Lee VW, Pang KK, Hui KC, Kwok JC, Leung SL, Yu DS, et al. Medication adherence: is it a hidden drugrelated problem in hidden elderly? Geriatr Gerontol Int 2013;13(4):978-85. semelhantes a essa, visando a educação em saúde e minimização dos fatores dificultadores da adesão ao tratamento.

Cabe ressaltar que a adesão ao tratamento medicamentoso não deve ser restrita às consultas médicas; antes, deve envolver a participação profissional multidisciplinar, atuando de forma integrada na abordagem da avaliação de risco, adoção de medidas de promoção da saúde e atendimento aos usuários dos serviços de acompanhamento ambulatorial.

Tais medidas são fundamentais para minimizar os gastos dos serviços de saúde de média e alta complexidade ${ }^{29}$ e, em especial, reduzir as incapacidades e agravos advindos da não adesão ao tratamento medicamentoso, promovendo melhor qualidade de vida aos idosos acompanhados ambulatorialmente.

7. Rajpura JR, Nayak R. Role of illness perceptions and medication beliefs on medication compliance of elderly hypertensive cohorts. J Pharm Pract 2014;27(1):19-24.

8. Calhoun DA, Jones D, Textor S, Goff DC, Murphy TP, Toto RD, et al. Resistant hypertension: diagnosis, evaluation, and treatment a scientific statement from the American Heart Association Professional Education Committee of the Council for High Blood Pressure, Research. Hypertension 2008;51(6):1403-19.

9. Ribeiro AQ, Rozenfeld S, Klein CH, César CC, Acurcio FA. Inquérito sobre uso de medicamentos por idosos aposentados, Belo Horizonte, MG. Rev Saúde Pública 2008;42(5):724-32.

10. Folstein MF, Folstein SE, McHugh PR. "Mini-mental state": a practical method for grading the cognitive state of patients for the clinician. J Psychiatr Res 1975;12(3):189-98.

11. Lourenço RA, Veras RP. Mini-Exame do Estado Mental: características psicométricas em idosos ambulatoriais. Rev Saúde Pública 2006;40(4):712-19.

12. Han C, Jo SA, Jo I, Kim E, Park MH, Kang Y. An adaptation of the Korean mini-mental state examination (K-MMSE) in elderly Koreans: demographic influence and population-based norms (the AGE study). Arch Gerontol Geriatr 2008;47(3):302-10. 
13. Valle EA, Castro-Costa E, Firmo JOA, Uchoa E, Lima-Costa MF. Estudo de base populacional dos fatores associados ao desempenho no Mini Exame do Estado Mental entre idosos: Projeto Bambuí. Cad Saúde Pública 2009;25(4):918-26.

14. Katz S, Ford AB, Moskowitz RW, Jackson BA, Jaffe MW. Studies of illness in the aged the index of ADL: a standardized measure of biological and psychosocial function. JAMA 1963;185(12):914-9.

15. Delgado AB, Lima ML. Contributo para validação concorrente de uma medida de adesão aos tratamentos. Psicol Saúde Doenças 2001;2(2):81-100.

16. Bloch KV, Melo AN, Nogueira AR. Prevalência da adesão ao tratamento anti-hipertensivo em hipertensos resistentes e validação de três métodos indiretos de avaliação da adesão. Cad Saúde Pública 2008;24(12):2979-84.

17. Bastos-Barbosa RG, FerriolliI E, MorigutiI JC, NogueiraI CB, NobreI F, Ueta J, et al. Adesão ao tratamento e controle da pressão arterial em idosos com hipertensão. Arq Bras Cardiol 2012;99(1):23-31.

18. Flores LM, Mengue SS. Uso de medicamentos por idosos em região do sul do Brasil. Rev Saúde Pública 2005;39(6):924-9.

19. Loyola Filho AI, Uchoa E, Lima-Costa MF. Estudo epidemiológico de base populacional sobre uso de medicamentos entre idosos na Região Metropolitana de Belo Horizonte, Minas Gerais, Brasil. Cad Saúde Pública 2006;22(12):2657-67.

20. Instituto Brasileiro de Geografia e Estatística. Indicadores sociodemográficos e de saúde no Brasil 2009. Rio de Janeiro: IBGE; 2009 [acesso em 5 fev 2014]. Disponível em: www.ibge.gov.br

21. Galato D, Silva ES, Tiburcio LS. Estudo de utilização de medicamentos em idosos residentes em uma cidade do sul de Santa Catarina (Brasil): um olhar sobre a polimedicação. Ciênc Saúde Coletiva 2010;15(6):2899-905.

22. Faria HTG. Fatores relacionados à adesão do paciente diabético à adesão terapêutica medicamentosa [dissertação]. Ribeirão Preto: Universidade de São Paulo, Escola de Enfermagem de Ribeirão Preto; 2008.

23. Rocha CH, Oliveira APS, Ferreira C, Faggiani FT, Schroeter G, Souza ACA, et al. Adesão à prescrição médica em idosos de Porto Alegre, RS. Ciênc Saúde Coletiva 2008;13(1):703-10.

24. Henriques MAP. Adesão ao regime terapêutico em idosos na comunidade: eficácia das intervenções de enfermagem [tese]. Lisboa: Universidade de Lisboa, Escola Superior de Enfermagem de Lisboa; 2006.

25. Carvalho ALM, Leopoldino RWD, Silva JEG, Cunha CP. Adesão ao tratamento medicamentoso em usuários cadastrados no Programa Hiperdia no município de Teresina (PI). Ciênc Saúde Coletiva 2012;17(7):1885-92.

26. Marin MJS, Cecílio LCO, Perez AEWUF, Santella F, Silva CBA, Filho JRG, et al. Caracterização do uso de medicamentos entre idosos de uma unidade do Programa Saúde da Família. Cad Saúde Pública 2008;24(7):1545-55.

27. Giatti L, Barreto SM. Saúde, trabalho e envelhecimento no Brasil. Cad Saúde Pública 2003;19(3):759-71.

28. Dourado CS, Macêdo-Costa KNF, Oliveira JS, Leadebal ODCP, Silva GRF. Adesão ao tratamento de idosos com hipertensão em uma unidade básica de saúde de João Pessoa, Estado da Paraíba. Acta Sci., Health Sci 2011; 33(1):9-17.

29. Girotto E, Andrade SM, Cabrera MAS, Matsuo T. Adesão ao tratamento farmacológico e não farmacológico e fatores associados na atenção primária da hipertensão arterial. Ciênc Saúde Coletiva 2013;18(6):1763-72. 